\title{
Colgajo de avance coronal y técnica de túnel usando matriz derivada del esmalte (EMD) en el tratamiento de recesiones gingivales múltiples: una revisión de la literatura
}

\author{
Eduardo David Peregrino Henríquez, ${ }^{*}$ Claudia Alejandra Guzmán Carranza, ${ }^{\ddagger}$ \\ Gerardo Mendoza Azpur§
}

\section{RESUMEN}

La recesión de la encía o tejido marginal gingival es denotada con base al desplazamiento del tejido marginal gingival hacia apical de la unión cemento-esmalte, exponiendo la superficie radicular. La influencia de factores (técnica de cepillado abrasiva, cepillo dental extremadamente duro, biotipo gingival delgado y trauma de oclusión secundario) desencadenan la pérdida de dicho tejido marginal, problemas funcionales y estéticos. En tal sentido, existen diversas técnicas quirúrgicas evocadas al tratamiento de la migración apical del tejido blando; de esta manera, el colgajo de avance coronal y la técnica de túnel son altamente predictibles, ya que muestran resultados favorables en términos de cobertura radicular. Los diversos estudios muestran una alta tasa de éxito para ambas técnicas, existiendo ausencia de revisiones en la utilización del EMD en el tratamiento de la cobertura radicular.

Palabras clave: Colgajo de avance coronal + EMD, técnica de túnel + EMD, recesiones gingivales.

\section{INTRODUCCIÓN}

La recesión de la encía o tejido marginal gingival es denotada con base al desplazamiento del tejido marginal gingival hacia apical de la unión cementoesmalte, exponiendo la superficie radicular al medio de la cavidad oral. ${ }^{1,2}$ La localización real del tejido

* Docente de Estomatología, Universidad Privada Antenor Orrego, Trujillo-Perú, RELAPED Research Group.

‡ Alumna de Estomatología, Universidad Privada Antenor Orrego, Trujillo-Perú.

$\S$ Director de la Especialidad de Periodoncia e Implantes, Universidad Científica del Sur, Lima-Perú.

Recibido: 21 de agosto de 2019. Aceptado: 12 de octubre de 2019.

Este artículo puede ser consultado en versión completa en www.medigraphic.com/periodontologia

\begin{abstract}
The recession of the gingiva or marginal gingival tissue is denoted based on the displacement of the marginal gingival tissue towards the apical of the cement-enamel junction, exposing the root surface. The present pathology presents a very diverse course, which is influenced by factors (abrasive brushing technique, extremely hard toothbrush, thin gingival biotype and secondary occlusion trauma). In this sense, there are various surgical techniques evoked to the treatment of soft tissue apical migration; in this way the Coronal Advance Flap is and the Tunnel technique are predictable technique, showing favorable results in terms of root coverage. The various studies show a high success rate for both techniques, in contrast, there are no reviews based on the use of EMD in the treatment of root coverage.
\end{abstract}

Keywords: Coronally advanced flap + EMD, tunnel technique + $E M D$, gingival recessions.

marginal corresponde al nivel de la inserción epitelial en la pieza dentaria, siendo la altura tentativa a nivel de cresta del margen gingival, en contraste con su posición real del presente margen o tejido gingival, determinando la gravedad de la recesión gingival. ${ }^{3}$

Es importante mencionar que dentro de la causalidad de las recesiones gingivales presentamos ciertos factores que incrementan o aquejan al paciente, tales como el incremento de la sensibilidad dentinaria, técnica de cepillado defectuosa e inconstante y el aspecto estético (como punto primordial que concierne en la actualidad a una gran cantidad de pacientes), son signos y síntomas característicos en cada uno de los que presentan este tipo de patología. ${ }^{4}$ Por otra parte, la presente condición se manifiesta clínicamente por la retracción de la encía marginal dirigida hacia la zona apical de la pieza o conjunto de piezas dentarias afectadas. En tal sentido, podemos apreciar diferentes 
Rev Mex Periodontol 2019; X (3): 65-68

lesiones clínicas presentes como abrasión, abfracción y caries radicular en dicha zona afectada. ${ }^{5}$

En tal sentido, los diferentes estudios epidemiológicos datan a la prevalencia de recesiones gingivales en fluctuaciones, las cuales oscilan alrededor de 50\% (en Norteamérica), en contraste con otros países como Brasil que estima $83.4 \%$, Chile presenta $68 \%$ y finalmente Perú presenta una tasa de $72.9 \%$. De la información mencionada, podemos observar la variación de datos en relación a los presentes estudios realizados en diversas partes de Sudamérica. ${ }^{6,7}$

En este contexto, Miller (1985) recalcó que el objetivo clínico primordial de todo procedimiento quirúrgico de recubrimiento radicular está basado en obtener naturalidad frente a los tejidos gingivales adyacentes, la disminución de la profundidad al sondaje (PS) y la ganancia de adherencia clínica. ${ }^{8}$

$\mathrm{Al}$ respecto, el recubrimiento radicular a través de la técnica de avance o reposicionamiento coronal utilizada desde el año 1956, propuesta por Grupe y Warren, es considerada altamente predictible en la actualidad y en ella se estandariza el reposicionamiento marginal del tejido hallado apicalmente. ${ }^{9} \mathrm{La}$ presente técnica tiene como finalidad el reposicionamiento del tejido blando (encía) en la unión cementoesmalte (UCE), devolviendo al paciente a un estado de cobertura radicular completa. ${ }^{10}$ De manera adicional, podemos apreciar clínicamente el uso del colgajo de avance coronal (CAF) en conjunto con matriz derivada de esmalte (EMD), lo que evidencia un mejor resultado en temas de predictibilidad en el tiempo. ${ }^{11}$

En contraste, la técnica de túnel (TUN) propuesta y descrita por Allen A (1994) y luego modificado por Allen E y Cummings (2002), en los últimos años ha incrementado su selección en la toma de decisiones clínicas por parte del especialista en el ámbito de la periodoncia, debido a su alta predictibilidad como promesa de un tratamiento correctivo y estético en el tratamiento de la migración apical del margen gingival (recesiones gingivales). ${ }^{12,13}$

De las evidencias mencionadas, apreciamos diferentes estudios que muestran la eficacia y efectividad de la presente técnica en conjunto con la utilización de la matriz derivada de esmalte (EMD). ${ }^{14,15}$ En contraste, el colgajo de avance coronal presenta mayores estudios en cuanto a su eficacia y predictibilidad utilizando diversos injertos y mejorando sus resultados a corto y largo plazo. ${ }^{16}$

La mayor parte de la revisión, se centra en evaluar la predictibilidad (tasa de éxito a largo plazo) en los diversos procedimientos de recubrimiento. La mayor cantidad de revisiones y de resultados favorables aportados hoy en día y con éxito a largo plazo le pertenecen al colgajo de avance coronal con la utilización de EMD en 92\%, en comparación con la técnica de túnel, la cual ofrece $82 \% .{ }^{17,18}$

En tal sentido, las técnicas antes mencionadas han demostrado elevadas tasas de éxito. De esta forma, la utilización de injertos y sustancias para mejorar su efectividad a largo plazo es sin lugar a dudas la mejor herramienta a elegir en el éxito de la regeneración periodontal. ${ }^{19}$ La utilización de EMD, como regenerador periodontal, puede atribuirse a la influencia sobre las células presentes en la proliferación de tejidos (duro y suave) y los diversos factores angiogénicos, favoreciendo el proceso de cicatrización. ${ }^{20,21}$

De las evidencias expuestas anteriormente, la finalidad de la presente revisión es recapitular la mejor evidencia disponible en cuanto a la elección entre ambas técnicas quirúrgicas mencionadas utilizando la matriz derivada de esmalte (EMD), evaluando la predictibilidad de ambos tratamientos y la tasa de éxito en el recubrimiento radicular completo en el tratamiento de pacientes con recesiones gingivales múltiples.

\section{AnTECEDENTES}

En el estudio realizado por Zuhr O y colaboradores (2014), analizaron los resultados clínicos luego de someterse a procedimientos quirúrgicos de cobertura radicular (técnica de túnel en comparación al colgajo de avance coronal utilizando matriz derivada de esmalte) en 24 pacientes con recesiones gingivales localizadas. Adicionalmente, se realizó en 47 lugares afectados con recesiones gingivales Miller tipo I y II y se evaluaron los resultados a los seis y 12 meses postcirugía. Finalmente, se obtuvo resultados clínicos favorables para la técnica de túnel (TUN) en comparación con el colgajo de avance coronal (CAC). ${ }^{22}$

Por otro lado, en el estudio propuesto por VincentBugnas S y colegas (2015), evaluaron los resultados clínicos de la técnica de túnel, en el cual se cambió el uso de injerto de tejido conectivo (ITC) por la matriz derivada de esmalte (EMD) en el tratamiento de recesiones gingivales Miller tipo I. Se utilizaron 26 piezas dentarias afectadas en 14 pacientes, a quienes se les realizó la cirugía de recubrimiento radicular 
aplicando la técnica de túnel, los aspectos clínicos a evaluar fueron la profundidad al sondaje, nivel de adherencia clínica y el grosor de tejido queratinizado. De las evidencias anteriores, se obtuvo a manera de alternativa el uso de EMD en lugar del injerto de tejido conectivo, visualizando resultados clínicos en cuanto a la cobertura radicular y la disminución de la morbilidad en la toma del injerto del paladar y evitando molestias postquirúrgicas. ${ }^{23}$

\section{Conclusiones}

La revisión nos permite concluir:

- La mayor cantidad de revisión y de resultados favorables aportados a la actualidad y con éxito a largo plazo (predictibilidad) le pertenecen al colgajo de avance coronal con la utilización de EMD en $92 \%$ en comparación con la técnica de túnel, la cual ofrece $82 \% .{ }^{19}$

- Adicionalmente, es importante mencionar a las modificaciones existentes en la actualidad, las cuales se han desarrollado últimamente (colgajo a espesor parcial y el aprestamiento desde un punto de vista microquirúrgico) al tener como objetivo principal mejorar los resultados finales. ${ }^{20}$

- Finalmente, el uso de EMD brinda a ambas técnicas quirúrgicas un mayor desarrollo debido a sus compuestos, los cuales sirven de proteínas con una alta capacidad para formar fibras de ligamento periodontal, cemento radicular y tejido óseo. ${ }^{21}$

\section{REFERENCIAS}

1. Kassab MM, Cohen RE. The etiology and prevalence of gingival recession. J Am Dent Assoc. 2014;134 (2): 220-225.

2. Chrysanthakopoulos N. Gingival recession: prevalence and risk indicators among young greek adults. J Clin Exp Dent. 2014; 6 (3): 243-249.

3. Mythri S, Arunkumar SM, Hegde S, Rajesh SK, Munaz M, Ashwin D. Etiology and occurrence of gingival recession. J Indian Soc Periodontol. 2015; 19 (6): 671-675.

4. Guttiganur N, Aspalli S, Sanikop MV, Desai A, Gaddale R, Devanoorkar A. Classification systems for gingival recession and suggestion of a new classification system. Indian J Dent Res. 2018; 29: 233-237.

5. Heasman PA, Holliday R, Bryant A, Preshaw PM. Evidence for the occurrence of gingival recession and non-carious cervical lesions as a consequence of traumatic toothbrushing. J Clin Periodontol. 2015; 42 (Suppl. 16): S237-S255. doi: 10.1111/jcpe. 12330 .

6. Rebele SF, Zuhr O, Schneider D, Jung RE, Hürzeler MB. Tunnel technique with connective tissue graft versus coronally advanced flap with enamel matrix derivative for root coverage: a RCT using 3D digital measuring methods. Part II. Volumetric studies on healing dynamics and gingival dimensions. J Clin Periodontol. 2014; 41 (6): 593-603.

7. Vincent-Bugnas S, Charbit Y, Lamure J, Mahler P, Dard MM. Modified tunnel technique combined with enamel matrix derivative: a minimally invasive treatment for single or multiple class I recession defects. J Esthet Restor Dent. 2015; 27 (3): 145-154.

8. Miller P. A classification of marginal tissue recession. Int J Periodontics Restorative Dent. 1985; 4 (2): 8-13.

9. Grupe H, Warren R. Repair of gingival defects by a sliding flap operation. Journal of Periodontology. 1956; 27: 92-95.

10. Allen AL. Use of the supraperiosteal envelope in soft tissue grafting for root coverage. II. Rationale technique. Int $\mathrm{J}$ Periodontics Restorative Dent. 1994; 14: 302-315.

11. Allen EP, Cummings LC. The role of periodontal plastic surgery in esthetic dentistry. Tex Dent J. 2002; 119, 1008 1015.

12. Restrepo O. Coronally repositioned flap: report of four cases. J Periodontol. 1973; 44: 564-567.

13. Miron RJ, Sculean A, Cochran DL, Froum S, Zucchelli G, Nemcovsky $\mathrm{C}$ et al. Twenty years of enamel matrix derivative: the past, the present and the future. J Clin Periodontol. 2016; 43 (8): 668-683. https://doi.org/10.1111/jcpe.12546.

14. Potârnichie O, Solomon S, Păsărin L, Mârțu A, Nițescu DC, Mârțu S. Statistical study on the prevalence of gingival recession in young adults. International Journal of Medical Dentistry. 2013; 3 (3): 219-224.

15. Zucchelli G, Tavelli L, Barootchi S, Stefanini M, Rasperini G, Valles $\mathrm{C}$ et al. The influence of tooth location on the outcomes of multiple adjacent gingival recessions treated with coronally advanced flap: a multicenter re-analysis study. J Periodontol. 2019; 90 (11): 1244-1251.

16. Cairo F, Nieri M, Pagliaro U. Efficacy of periodontal plastic surgery procedures in the treatment of localized facial gingival recessions. A systematic review. J Clin Periodontol. 2014; 41 (Suppl 15): S44-62.

17. Rebele SF, Zuhr O, Schneider D, Jung RE, Hürzeler MB. Tunnel technique with connective tissue graft versus coronally advanced flap with enamel matrix derivative for root coverage: a RCT using 3D digital measuring methods. Part II. Volumetric studies on healing dynamics and gingival dimensions. J Clin Periodontol. 2014; 41: 593-603. doi: 10.1111/jcpe.12254.

18. Zuhr O, Rebele SF, Schneider D, Jung RE, Hürzeler MB. Tunnel technique with connective tissue graft versus coronally advanced flap with enamel matrix derivative for root coverage: a RCT using 3D digital measuring methods. Part I. Clinical and patient-centred outcomes. J Clin Periodontol. 2013; 41: 582-592. doi: 10.1111/ jcpe.12178.

19. Sculean A, Cosgarea R, Stähli A, Katsaros C, Arweiler NB, Miron RJ et al. Treatment of multiple adjacent maxillary Miller class I, II, and III gingival recessions with the modified coronally advanced tunnel, enamel matrix derivative, and subepithelial connective tissue graft: a report of 12 cases. Quintessence Int. 2016; 47 (8): 653-659.

20. Santamaria MP, Neves FL, Silveira CA, Mathias IF, Fernandes-Dias SB, Jardini MA et al. Connective tissue graft and tunnel or trapezoidal flap for the treatment of single maxillary gingival recessions: a randomized clinical trial. J Clin Periodontol. 2017; 44 (5): 540-547. 
Rev Mex Periodontol 2019; X (3): 65-68

21. Tavelli L, Barootchi S, Nguyen TVN, Tattan M, Ravidà A, Wang HL. Efficacy of tunnel technique in the treatment of localized and multiple gingival recessions: a systematic review and a meta-analysis. J Periodontol. 2018; 89 (9):1075-1090.

22. Aydinyurt HS, Tekin Y, Ertugrul AS. The effect of enamel matrix derivatives on root coverage: a 12-month follow-up of a randomized clinical trial. Braz Oral Res. 2019; 33: e006.

23. Kobayashi E, Fujioka-Kobayashi M, Saulacic N, Schaller B, Sculean A, Miron RJ. Effect of enamel matrix derivative liquid in combination with a natural bone mineral on new bone formation in a rabbit GBR model. Clin Oral Implants Res. 2019; 30 (6): 542-549.

\section{Correspondencia:}

Eduardo David Peregrino Henríquez

E-mail: ursiget.rotante3@usil.edu.pe eduper23@hotmail.com 